\title{
Digital Imaging in the Radiation Oncology Environment: A Personal Computer Local Area Network Solution
}

\author{
Patrick M. Stafford, Eric E. Martin, James C.H. Chu, Bruce A. Davidson, and Gerald E. Hanks
}

\begin{abstract}
Personal computer $(\mathrm{PC})$ driven digital imaging devices are now becoming available in the market place for use in radiation oncology to produce what are known as real time portal images. These limited contrast images are used to verify the patient anatomy under treatment by megavoltage $x$-rays and are a vital part of the patient treatment. The current generation of devices can produce both single and movie loop images in real time for physician review and approval. To disseminate these images and other digital images used in the planning and delivery of radiation therapy, a PC-based picture archiving and communications system has been developed that is tailored to the special needs of radiation oncology.
\end{abstract}

Copyright $\odot 1991$ by W.B. Saunders Company

KEY WORDS: digital image, radiation oncology, PACS, real time portal image, computed tomography, magnetic resonance imaging.

A $S$ THE availability of digital imaging devices for radiation oncology increases, and with the use of digitally based images such as computerized tomography (CT) and magnetic resonance imaging (MRI) for treatment planning, the capability to distribute and display this information will become very important. Several devices are currently being marketed that allow the acquisition of digital portal images, and devices that will allow digital simulation and simulation-based CT imaging are now in the final stages of development. With this equipment, a three-therapy machine department that has two simulators would have five devices generating digital images on a daily basis. In the span of 1 year, these devices would generate well over 20,000 images in the course of treating approximately 1,200 patients. Add to this the burden of CT and MRI images for treatment planning, and the number of images to be handled in a year could easily exceed 50,000. With this large flow of images, the ability to distribute, manipulate, and store these images with minimal effort and financial expenditure is a necessity.

In a typical radiation oncology department, the simulation images must be viewed, fields shaped to protect normal tissues, and approved by the attending physician before the patient treatment can begin. Those images that have blocks added must be sent to a mold room to have the blocks cut. The treatment planning can be as simple as an external contour derived from a CT-simulation image or as complex as a noncoplanar three-dimensional treatment plan utilizing $40 \mathrm{CT}$ images with multiple internal contours. Once the therapy has begun, set-up and multiple weekly portal films must be taken to verify the radiation fields. With capability of real-time portal imaging, it is probable that some patients will have "movies" of their ports made to ensure that the treatment volume is not moving outside the treatment field with respiration or other movements, thus increasing the number of images taken by perhaps a factor of 10. Therefore, one can see that dosimetrists, block cutters, physicians, and physicists must have the capability to view, modify, and extract information from these images. There must be provision for the drawing of treatment field modifications, comments, and other information on the simulation and portal film images. Because these images are part of the patient's legal record, the modifications and approvals must be performed in a fashion that ensures that the attending physician has indeed reviewed and approved the images. The $\mathrm{CT}$ and MRI images must be contoured for internal normal and tumor anatomy, and this information must be passed to the treatment planning systems.

Due to the number of people and interactions associated with handling these images, the workstations must be readily available, and must be economical to provide the required number of display systems. We have decided upon the use of readily available personal computer technology, along with a commercially available local area network (LAN) to perform these func-

From the Fox Chase Cancer Center, The University of Pennsylvania, Philadelphia, and HandiSoft Corporation, Philadelphia, $P A$.

Address reprint requests to Dr Patrick M. Stafford, Department of Radiation Oncology, Fox Chase Cancer Center, Central and Shelmire Aves, Philadelphia, PA 19111.

Copyright (C) 1991 by W.B. Saunders Company

0897-1889/91/0402-0007\$03.00/0 
tions. The medical image display (MID) software is being developed in a collaborative effort with the HandiSoft Corporation (Philadelphia, PA) and Fox Chase Cancer Center (Philadelphia, PA) to perform these functions.

\section{LAN SYSTEM HARDWARE}

The overall hardware configuration of the LAN is shown in Fig. 1. The workstations for the MID system are eighter PC-compatible or Apple Macintosh (Cupertino, CA) computers linked via a thin wire Ethernet LAN in a bus configuration. The PC-compatible computers are Compaq $28612 \mathrm{MHz}$ (Houston, TX) compatibles with 4 Mbytes of memory, 16-bit super VGA resolution display cards and $35.6 \mathrm{~cm}$ color monitors. The Apple computers are either Macintosh SE-30 or II computers with 35.6 or $50.8 \mathrm{~cm}$ display color monitors, 8-bit color display cards and 8 Mbyte of random access memory. The workstations are located in each physicist, physician, and resident's office, in the conference rooms and library, and at selected areas within the department. The office machines were selected by the preference of the user for either PC or Macintosh environments. The server for the system is a Compaq 386/20 with both internal hard drives and a 1.2 Gbyte removable media, erasable optical disk. A streaming tape drive is provided for backup of the internal hard drives. The LAN is bridged by a Retix (Santa Monica, CA) gateway to the departmental micro VAX 3600, allowing transfer of data and images to and from the network using the TCP/IP data interchange protocol. The microVAX (Digital Equipment Corporation, Maynard, MA) contains a 660 Mbyte disk, 32 Mbyte of memory and a 6250 bits per inch

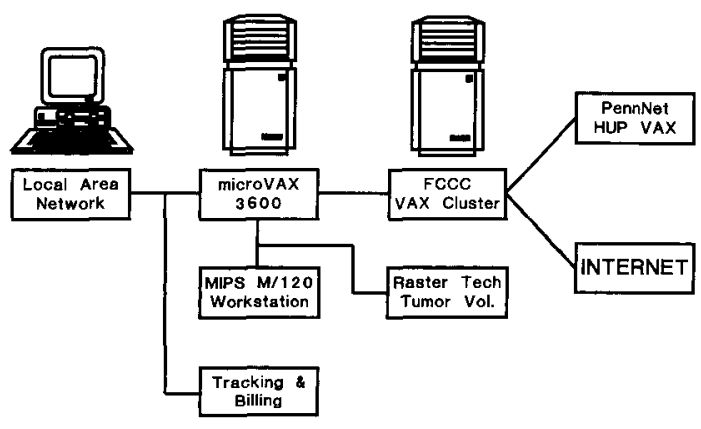

Fig 1. Overall hardware and systems configuration at Fox Chase Cancer Center.
$1.27 \mathrm{~cm}$ tape drive. The microVAX is linked to the Cancer Center VAX cluster consisting of five interlinked VAX/785 and 8800 mainframes, which are used for routine treatment planning calculations. A MIPS RS-2030/M-120 (MIPS Computer Systems, Sunnyvale, CA) reduced instruction set computer (RISC) is linked to the network to perform three-dimensional (3D) treatment planning calculations. The MIPS machine is populated with $16 \mathrm{Mb}$ of memory, a 600 $\mathrm{Mb}$ hard drive and a streaming tape drive. $\mathrm{A}$ Raster Technologies Model One/25 (Westford, MA) 24 bit graphics display is also available for graphics display and tumor volumetrics.

A conference room display system is now under development that will feature an array of five $50.8 \mathrm{~cm}$ color monitors driven by a Macintosh II/X computer and five 8-bit Apple color display cards. This hardware will allow the display of up to 10 stimulation and/or portal films at one time to facilitate the conference review of new patients and patients undergoing therapy.

Several hardcopy devices are available to the users of the LAN, including an HP-7475A six pen color plotter, a nine pin narrow format printer, a 24-pin wide format color printer, an Apple LaserWriter II/NTX Postscript laser printer, and for the digital images, a Lasertechnics 300D (Albuquerque, NM) continuous tone laser printer. The Lasertechnics printer has a resolution of $2048 \times 2900$ for a $20.3 \times 28.7 \mathrm{~cm}$ image (100 pixels $/ \mathrm{cm})$ with a dynamic gray scale of 256 levels. The printer can accommodate either paper or film to produce the hardcopy images. The printer is interfaced via a SCSI port, and produces a print in approximately 1.5 min from the time the image is disposed to be printed.

\section{IMAGING SYSTEM HARDWARE}

The imaging systems purchased for the department consist of a Fiber Imaging FiberVision (St Louis, MO) real time portal imaging device that produces a $480 \times 512$ pixel image with a maximum field size of $40 \times 40 \mathrm{~cm}$ at the radiation isocenter of the therapy machine. The images are produced by light from a scintillation screen transmitted by a bundle of fiber optics to a television camera. The output of the camera is "grabbed" by a video frame grabber installed in 
a Dell 80386 (Dell USA, Austin, TX) based PC, and the image is corrected for background and geometry effects by software at the $\mathrm{PC}$, producing an image with an 8-bit image depth. The PC is hooked by a standard Ethernet card into the LAN, allowing transfer of the images generated directly to the optical disk for storage. A pair of images produced by this system can be seen in Fig 2 as displayed by the MID software. The system is capable of producing an image every $1 / 30$ of a second, and are stored in random access memory (RAM) during the image acquisition phase. In practice, portal images are acquired every $1 / 4$ to $1 / 2 \mathrm{sec}$ to produce sufficient contrast with the megavoltage $\mathrm{x}$-rays being used. With 16 Mbyte of RAM, the system is capable of storing 55 images during a treatment.

The second system, to be delivered in August of 1990, is an S\&S Inficon Theraview system (Liverpool, NY) that uses a mirror system to reflect the image produced by a scintillation screen to a TV camera. The image is processed by an 80286 based central processing unit with a proprietary, high throughput image processing board, producing a $512 \times 512 \times 8$ bit image. The PC-compatible computer is interfaced to the LAN via a standard Ethernet card to allow image storage on the LAN optical disk drive. Image acquisition times are similar to that of the FiberVision system, allowing sequential still images at selected times during treatment, or "movie" images to be taken during therapy.

Current plans for simulation images are to digitize the simulation films via a laser scanning digitizer from ImageComm Systems. The scanner is driven by an AT compatible computer, which will again be interfaced to the networks by a standard Ethernet card. Specifications for new simulation and therapy equipment will require the installation of digital imaging equipment as part of the bid process at Fox Chase. With the planned replacement of a simulator and a linear accelerator in the next two years, it is anticipated that Fox Chase radiation oncol-

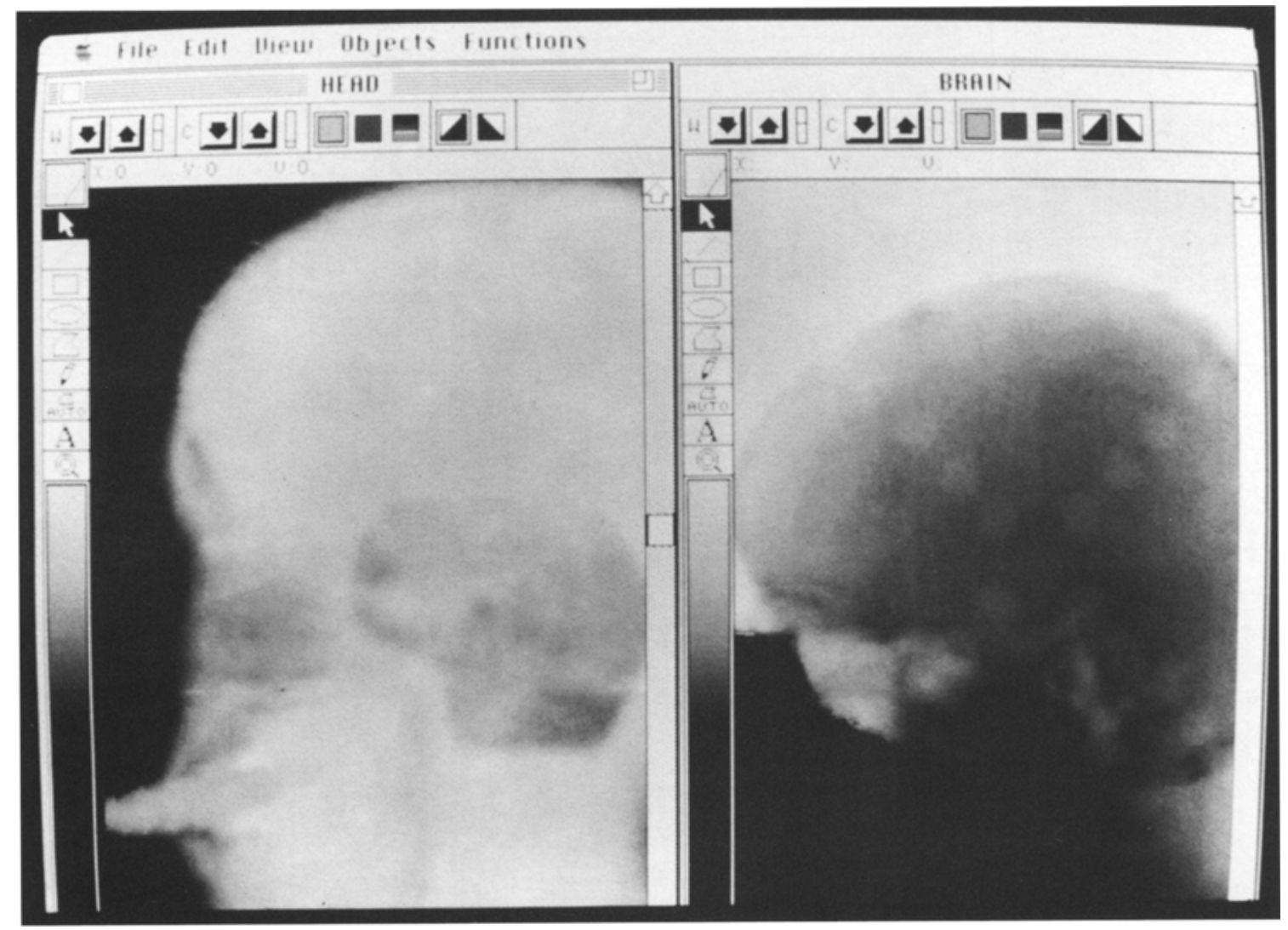

Fig 2. Real time portal images as displayed by MID on the Macintosh II/X workstation (Images courtesy of Fiber Imaging Corporation). 
ogy will be fully digital in its image production at that time.

\section{LAN SOFTWARE}

\section{Operating System}

The local area network operating system at Fox Chase is Novell Advanced Netware 2.15 (Novell Inc, Provo, UT) that allows both Macintosh and PC computers to share data and peripherals in a manner transparent to the user. Data is transmitted over this network using Ethernet at a rate of 10 Mbyte per sec. Software for tasks such as word processing and data management are handled by Microsoft Word and Microsoft EXCEL (Microsoft Corp, Redmond, WA), which allow users to interchange data with little effort on their part.

\section{Image Data Base}

The images generated on the LAN are an important part of the patient's legal record of therapy, and it is therefore important to ensure that the images retrieved are correctly identified. If modifications are made to the images, the modifications must be saved, and a record made of the attending physician making the modification. These modified images must then be saved in a read-only format to preserve the parts actually treated on the patient. Organization and retrieval of images is accomplished using Foxbase + (Fox Software, Inc, Perrysburg, $\mathrm{OH})$ a programmable data base management system by Fox Software. Foxbase+ was chosen because compilers are available for both PC and Macintosh platforms, allowing the data base structure to be compiled independently of the computer architecture. Flexible processing and sorting of images is programmed into the physician/data base interface, so that a physician can retrieve any active patient's images for use in the MID software, or by default only his active patient images will be made available. By clicking on the patient name and then on "radio buttons," a physician can select both the modality $(\mathrm{CT}$, simulation, real time portal images (RTPI) or MRI) and the view if the image is a planar one (right lateral, anterior, etc). Images can also be filtered via date with an "all before" or "all after" date, and multiple filters are supported. Access to the images is protected by two passwords, and an additional password is required to approve and/or modify the image for patient treatment. Once the images are approved, the MID toolbox is turned off for those images, preventing further modification of the images.

\section{Medical Image Display Software}

The MID software in its present operating form has been developed over the past 2 years in a collaborative effort by Fox Chase Cancer Center, and the HandiSoft Company. Because of the powerful image handling capabilities and the graphical user interface (GUI) available in Macintosh computer systems, the initial software development has been performed on this machine. A parallel effort for PC based systems has been instigated using Windows 3.0 as the GUI, which will ultimately duplicate the capabilities of the Macintosh software. The images displayed in this paper, and the capabilities described are currently implemented on the Macintosh platform.

Figure 3 illustrates the anticipated image and data flow throughout the LAN, with images originating within the department at the linear accelerators and simulator machines (A). These images are disposed directly to the optical disk drive with software written for each imaging device to allow storage of the image information in American College of Radiation-National Electrical Manufacturers Association format. ${ }^{1}$ Other images such as CT and MRI originate from outside radiation oncology, and are accessible on the VAX cluster. These images are downloaded through the departmental VAX, through the bridge and onto the LAN optical

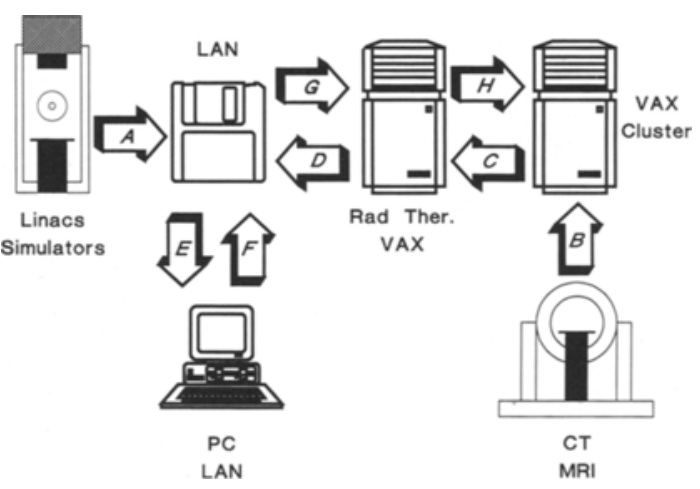

Fig 3. Departmental image flow from RTPI, simulation, CT, and MRI to the LAN. 
disk for active patients (B,C,D). Portal and simulation images are reviewed at the PC workstations, and modified and/or approved images are again stored on the optical disk (E,F). Similarly, for treatment planning, CT or MRI images may be contoured on the workstation, and these contours can then be sent to the VAX or MIPS machines for treatment planning calculations $(E, F, G)$. The isodose distributions can then be reviewed on the workstations by transfer from the VAX to the LAN (D,E). After end of treatment, patient portal and simulation images are archived to the VAX cluster to maintain working space on the LAN optical disk $(\mathrm{G}, \mathrm{H})$.

Referring to Fig 2, two portal images from the FiberVision portal imaging device are shown, the left image is a double exposure of the head and neck region with the radiation port shown in darker contrast. The right image is that of a whole brain irradiation. Along the top of the images, the FILE menu allows selection of disk drives available to the system, the image selection scroll box, and the image format scroll box. By pointing and clicking on the image name and format, the appropriate image can be displayed on the screen, or the images will automatically be retrieved in correct format using a file transfer link from the data base interface to MID. Multiple images can be activated, although in practice, two portal or simulation images or four CT or MRI images are a maximum for readability. Once the images are retrieved, the contrast level and window width can be varied by pointing and clicking on the up or down arrows in the $\mathrm{C}$ or $\mathrm{W}$ boxes. The small graph on the top of the left-hand column displays a graphical representation of where these thresholds are set. Next to the contrast adjustment box is the display format box that allows the user to choose (from left to right), the black and white conventional display, a green and black display (the eye is most sensitive to the green wavelengths of the spectrum), and a false color display. Next to these are the boxes for white on black or black on white display of the image (image inversion). Again the selections are made by clicking on the appropriate box. Below the contrast/window width graph are the click boxes for the tools available in MID. The arrow box converts the pointer from the cross-hair for auto contouring or the magnifying glass for magnification of the image, thus the cursor shape indicates the current function selected.

The square and ellipse boxes allow these objects to be drawn and sized interactively with the mouse in the overlay, using red lines.

The polygon tool allows irregularly shaped objects to be approximated by line segments of any length, by clicking the mouse at appropriate points around the margin of the object. Figure 4 shows a block modification performed in this fashion.

The pencil tool allows the user to continuously draw freehand a contour in the overlay, again by use of the mouse.

By clicking on the Auto box, the automatic contouring feature is engaged, allowing the user to set the autocontour level by using a variable region, sliding scale (set by the mouse) that turns the anatomy interface that is to be contoured a deep blue. For example, if the skin is to be autocontoured, the region surrounding the skin is set by the level controls to turn the air CT numbers to indicate deep blue. The cursor is then placed near the skin surface, and the mouse is clicked to begin the contouring process. By varying the level and width of the autocontouring control, bone, air and soft tissue interfaces such as spinal cord and kidney can easily be contoured. Figure 5 shows a CT slice with several internal organs and the skin contoured in this fashion.

The A box allows the user to enter text for the identification of contours as in Fig 5, or for comments as shown in Fig 4. The text becomes part of the contour file, and is saved with the contour information and image.

The magnifying glass box allows the user to selectively magnify a region of the image by $200 \%$ or $400 \%$ by placing the glass in the region desired and clicking the mouse button. One click is $200 \%$, and 2 clicks gives a $400 \%$ magnifcation. Figure 4 shows a portal image magnified $200 \%$ in this fashion. Clicking on the magnify box decreases the magnification by a factor of two for each successive click.

Currently in development is the distance measurement (ruler) tool, which will calculate the distance between two points indicated by the mouse, and an eraser tool, which will allow 


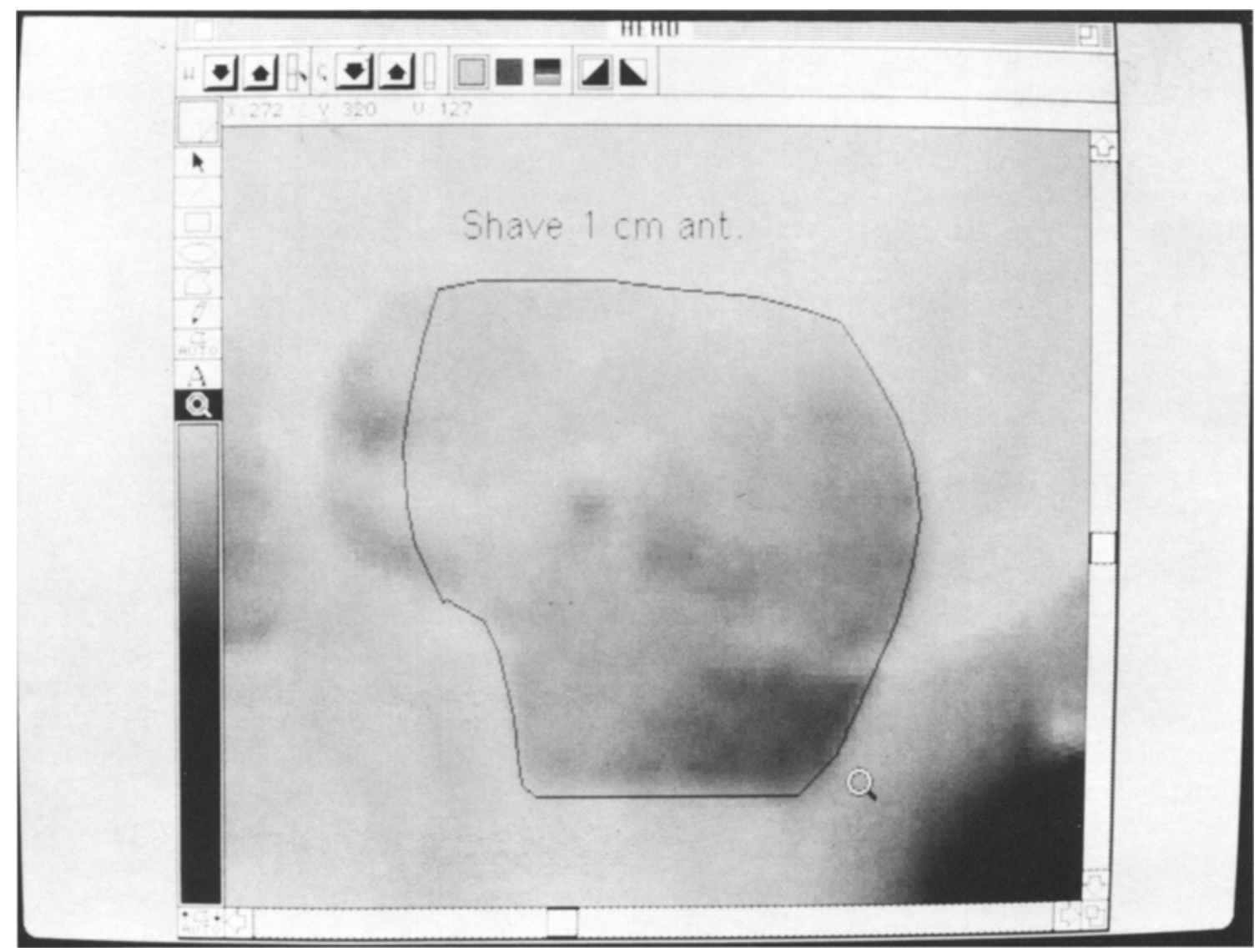

Fig 4. A $200 \%$ magnification of the left hand image of Fig 2 showing block modifications and textual information.

the contour to be selectively edited. A step-off tool is also to be developed that will symmetrically expand a region by a specified margin in centimeters.

Other capabilities of MID are provided by the EDIT menu, which allows UNDO, CUT, COPY and PASTE of contour and textual information. In this menu, one can also FLIP the image vertically and horizontally as well to allow the user to orient the image to suit his needs. Because patients are not always perfectly aligned in a CT or MRI scanner, some skin or other anatomy may be cut off by the reconstruction radius. To allow autocontouring of the skin in particular, the image cannot be contiguous with the edge of the reconstruction matrix, and so a NUDGE feature is provided. With this feature, one can move the image left, right, up or down by one or more pixels, substituting air value pixels in place of the displaced pixels.

The VIEW menu allows the scale size to be set manually for the image, or to visualize the contour/text overlay only, the image only, or a combined image.

The OBJECTS menu allows the user to set the font, size, style and alignment of the text used in the image.

The FUNCTIONS menu allows the manual setting of the autocontour level, giving a digital value for the upper and lower range of the contour level.

To allow the rapid review of a series of images, as in a portal image movie, provision is made to automatically open a series of images that have the same filename and a serial extension when the image is initially opened. When the image window is displayed, two additional click buttons are provided, to the right of the display format box. These buttons allow the user to flip through the image sequence as rapidly as he or she desires, and also provides a movie capability. The movie of the image sequence is started by holding down the SHIFT key and clicking on the down-pointing button. 
Fig 5. A CT slice as displayed by MID showing autocontoured skin and normal tissues. Note contour identifiers.

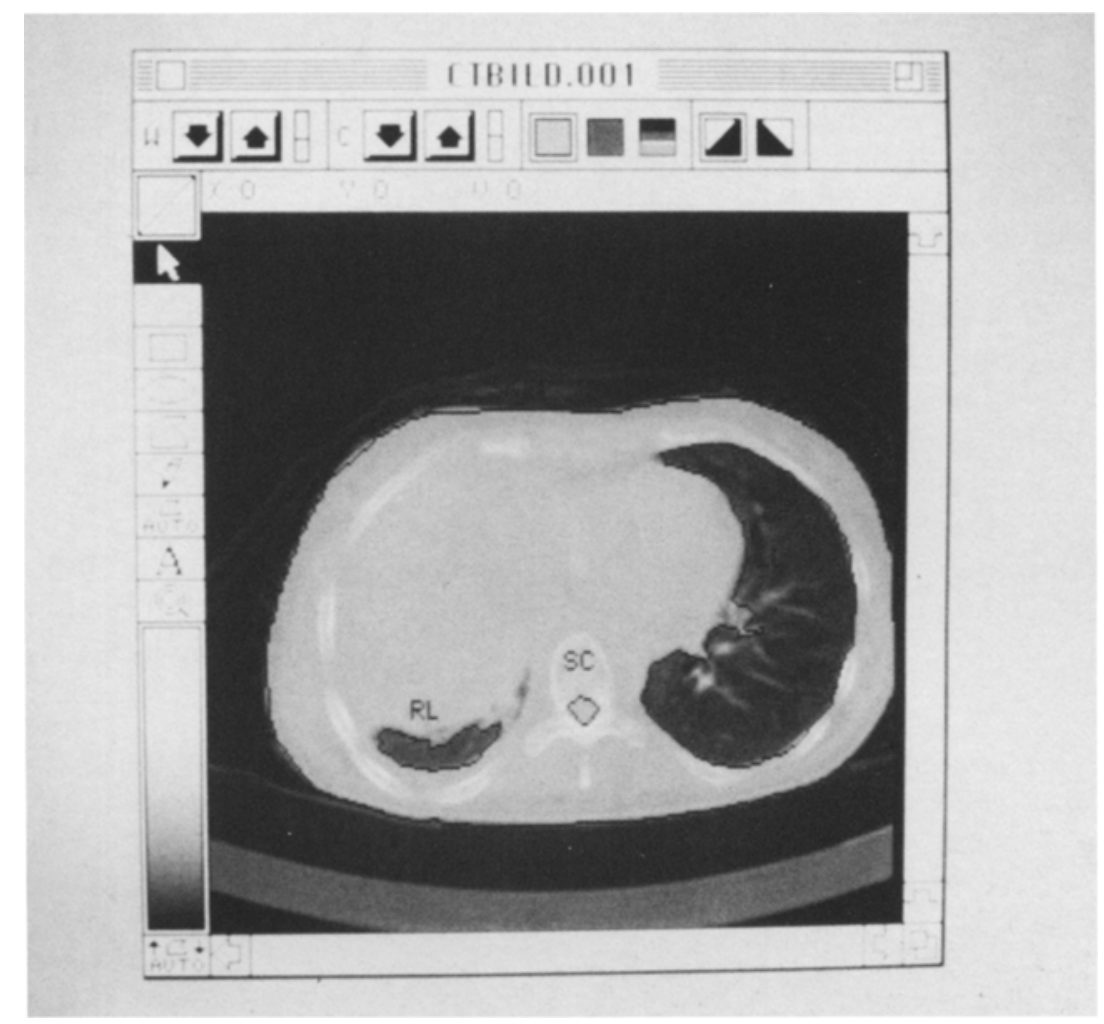

The speed of the movie review is varied by pressing the up or down arrow keys.

Because the portal and simulation images are part of the patients legal treatment record, a system has been devised based upon passwords to ensure that radiation ports or modifications of these ports are performed by only the attending physician staff. Two additional click boxes will appear on these images; APPROVED and MODIFY. When a portal or simulation image is in any way modified with field blocks, these contours can only be saved by clicking the MODIFY box. When this box is clicked, the user is prompted for a password that identifies the attending physician and places his or her name on the image header with a time and date stamp. This image is then saved as a read-only image with a later date and time stamp than the original, which is also retained. In development is the transfer of these block contours to the Par Scientific A/S ACD-4 (Par Scientific A/S, Denmark) computer driven block cutter, to automatically cut the block molds from plastic foam.

Other developments for the LAN as part of the information available to users will include data from the Varian RMS-2000 (Varian Medi- cal Equipment, Palo Alto, CA) record and verify system for each patient such as current total number of ports treated, total dose, etc. This system will use IBM PS/2 Model 80 PCs to transmit data from the simulators to the treatment machines on the LAN concerning field size, gantry angles, energy, etc and will verify these settings prior to each treatment. As patients are treated, it will also acquire data concerning each treatment as mentioned above, and will provide an interface to allow access to this data.

As the primary functions of image handling are accomplished with the MID system, other software which will interface with the dosimetry system will be developed, allowing the electronic review of dose distributions from either $2 \mathrm{D}$ or $3 \mathrm{D}$ calculations.

\section{CONCLUSIONS}

The success with which we have been able to use current PC technology to accomplish the nominal capabilities of a picture archiving and communications system has been very encouraging. In generating software that is tailored to the requirements of radiation oncology we will also 
have been able to extend these capabilities to streamline image handling, and to automate the sequence of events necessary to the production of blocks and approval of simulation and portal images. Because of the use of PC technology for the production of digital images from simulators and therapy machines, it is logical to use PC technology to display the resulting images. With the ability to use sophisticated autocontouring algorithms, the use of 3D virtual simulation and treatment planning will become less time consuming and a more useful clinical tool. With over $\$ 45,000.00$ per year spent on film, developer, and film storage at Fox Chase, one can repay the investment in hardware and software in approximately 7 to 10 years. More impor- tantly, the system allows: (1) greater ability to assure the quality of therapy without slowing throughput of patients; (2) the treatment of special situations not possible with existing technology and; (3) the computers are very helpful in other aspects of clinical research ongoing in the department and are useful therefore in other applications than just those of image display.

To summarize, we believe that the technology of PC LAN with currently available software can provide radiation oncology with a useful tool to perform not only PACS functions, but provide information and data services unique to the discipline.

\section{REFERENCES}

1. National Electrical Manufacturers Association: Digital Imaging and Communications. Washington, DC, Na- tional Electrical Manufacturers Association, 1988 (ACRNEMA 300-1988) 